\title{
Integration of textile-based sensors and Shimmer for breathing rate and volume measurement.
}

\author{
Carlos Rovira, Shirley Coyle, Brian Corcoran, \\ Dermot Diamond. \\ CLARITY - Centre for Sensor Web Technologies, \\ National Centre for Sensor Research, \\ Dublin City University Dublin 9, Ireland.
}

\author{
Florin Stroiescu, Kieran Daly. \\ Shimmer Research, \\ The Realtime Building, Clonshaugh Technology Park, \\ Dublin 17, Ireland.
}

\begin{abstract}
This paper present the characterisation of piezoresistive fabric sensors for measuring breathing patterns. While it has been shown that these sensors can be used to measure breathing rate with good reliability, breathing volume measurements are more complex due to drift and non-linear behaviour. Breathing patterns are extremely useful for a number of applications related to personal health and exercise monitoring. For example, breathing exercises form an essential part in the treatment of respiratory illnesses while in sports, breathing technique can help to improve the performance of athletes.
\end{abstract}

\section{INTRODUCTION}

The characterisation of piezoresistive fabric sensors for measuring body movements is important for the rapidly developing area of wearable sensors. In this paper we present the suitability of these sensors to monitor breathing patterns. While it has been shown that these sensors can be used to measure breathing rate with good reliability, breathing volume measurements are more complex due to drift and non-linear behaviour. To develop a model of sensor behaviour would improve the accuracy of measurements. This would be very useful for both clinical and athletic fields. For example, breathing exercises form an essential part in the treatment of respiratory illnesses while in sports, breathing technique can help to improve the performance of athletes $[1,2,3]$. The wearable, wireless Shimmer [4] platform, is being used to capture and process all the data from the sensors.

\section{METHODS}

This study uses a mechanical rig, shown in figure. 1, to perform rigorous testing of three piezoresistive fabrics (figure 2) acquired from Eeonyx (Eeonyx Corporation, Pinole, CA 94564, USA). The fatigue machine permits accurate testing for long periods of time simulating ribcage movements. Tests were performed to investigate the temporal response of the fabric sensors, the effects of applying pre-tension to the fabric and the drift of the sensor response over a number of hours. The responses of the sensors were compared after a different kind of test using the fatigue machine to examine the effect of sensor drift over time. Such characterisation is needed to optimise signal processing of on-body fabric sensors and also

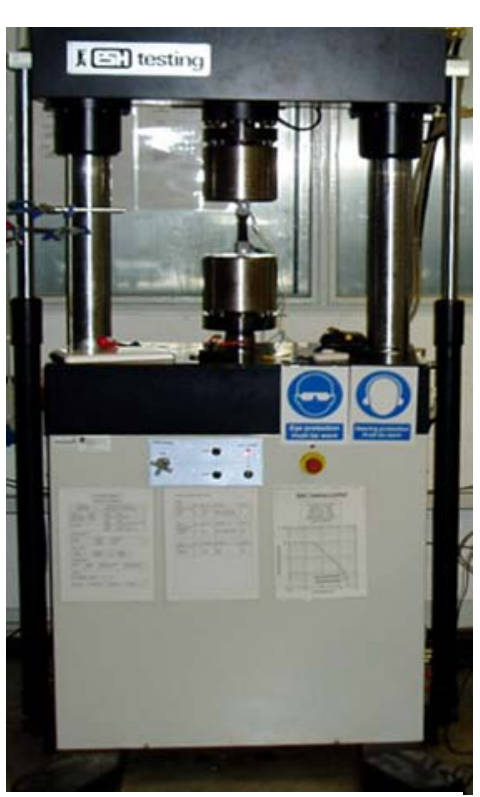

Figure 1. Sensor stressed by fatigue machine and connected to the Shimmer platform. to gain an understanding of the behaviour of different piezoresistive textile structures.

These sensors have electrical characteristics that permit measurement of the change in resistance in the different sensors. The Shimmer platform permits precise measurement of the sensor resistance. The sensor connected to the Shimmer device is stressed by the fatigue machine.

Shimmer is a small sensor platform well suited for wearable applications. Data are acquired using a 12 bit $\mathrm{A} / \mathrm{D}$ converter connected to an ultralow power consumption processor and at the same time, data are sent to a nearby laptop via Bluetooth. Shimmer Research's graphical display software initiates communication and shows the value of the resistance in real time. After this, data analysis and further signal processing is performed using Matlab.

\section{RESULTS}

Repeating the same experiment with the three materials exhibited the different response of each material. Each material operated within a different range of resistance different (fiigure 2) and consequently we had to adapt the ADC range for each sensor. The brown sensor has found to have the same input impedance of the ADC (the megaohm range $M \Omega$ ), but with the Grey and Black sensors we just need to adapt the different resistances from the amplifier, before de $\mathrm{ADC}$, for receiving data correctly. 


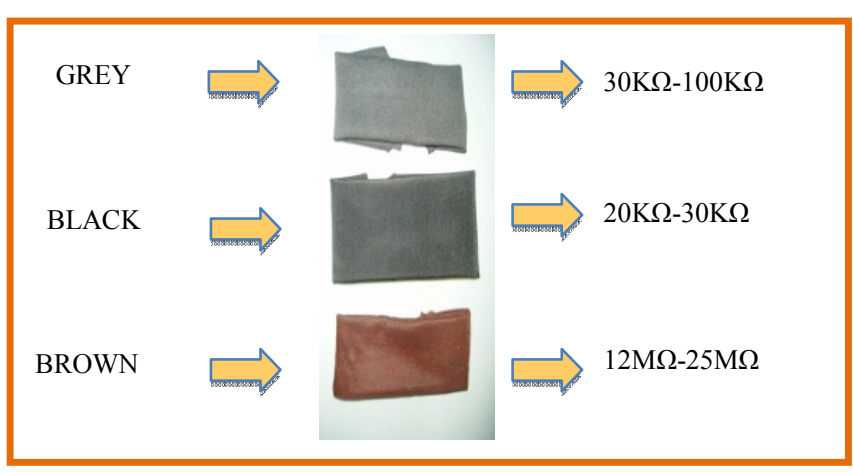

Figure 2. Three different piezoresistive fabrics.

Fig. 3 shows the results of an experiment where the change in extension of the Black sensor, is similar to the strain it would endure during breathing when worn on the body $(2 \mathrm{~mm})$. Fig. 3 shows the fabric (Black Sensor) due to expansion and contraction at rate $0.143 \mathrm{~Hz}$ from a starting extension of $28 \%$ of the fabric's initial length to a final extension of $32 \%$ of the initial fabric length.

From this data it is clear that the sensor can detect movements accurate to $0.1 \mathrm{~mm}$ precision which means that we can get information from small movements from the body. If we compare two different textile piezoresistive materials, the response of each is different but, after applying a Butterworth filter ( $\mathrm{fc}=0.4 \mathrm{~Hz}$ and $\mathrm{N}=7$ ) to reduce noise, both are suitable for measuring breathing movements. Pre-tension of the sensor is important because below a pre-tension level the material does not behave in a linear manner. The three fabrics were found to have different limits for pre-tension: $10 \%$ for black sensor, $25 \%$ for Grey sensor and 30\% for Brown sensor.



Grey Sensor test zoom

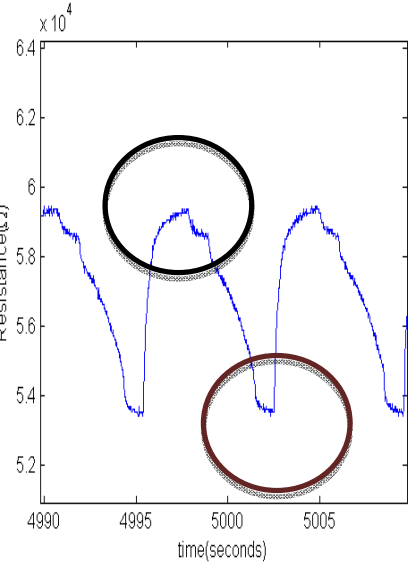

Black Sensor test zoom
Figure 4. Difference between Black and Grey sensor if the piezoelectric fabric is not pre-tensioned.

If we do the same test for a long period of time (more than 2 hours), and after repeating more than 10 times the same experiments, we come to the conclusion that we can predict how will respond the different textile sensors in each instance. Fig. 5 has an example of the response of the black sensor after a period of time of two and a half hours.

Fig. 6 shows the response of the grey sensor-following the same test procedure. The test was carried out for two and a half hours. The size of the sensor is the same and the conditions of the test as well. Analyzing the results we can see that the time response is different. But the important point is that by characterizing the sensors in this way we can predict this effect.
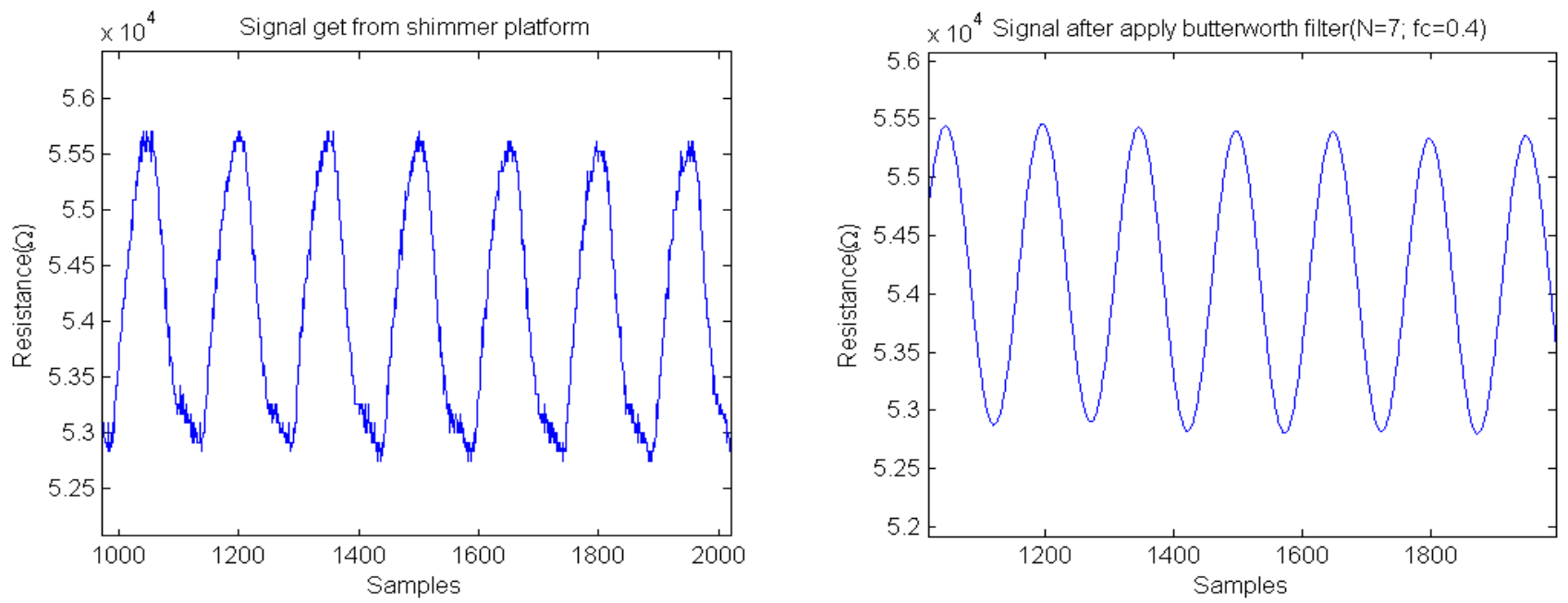

Figure 3. Data received from Black sensor; Extension: 2mm; cycles: 7; Figure 3a.-Raw signal received from Shimmer platform. Figure 3b.-Signal after application of Butterworth filter. 
Size sensor :

Lenght: $55 \mathrm{~mm}$

Width: $20 \mathrm{~mm}$

Textil sensor :Black

Initial length material: $65 \mathrm{~mm}$

Final length material $=75 \mathrm{~mm}$

Extension: $10 \mathrm{~mm}$

Sample: 50 sps

Time test: $2,5 \mathrm{~h}$

Frequency: $0.142 \mathrm{hz}$

Total cycles: 1200

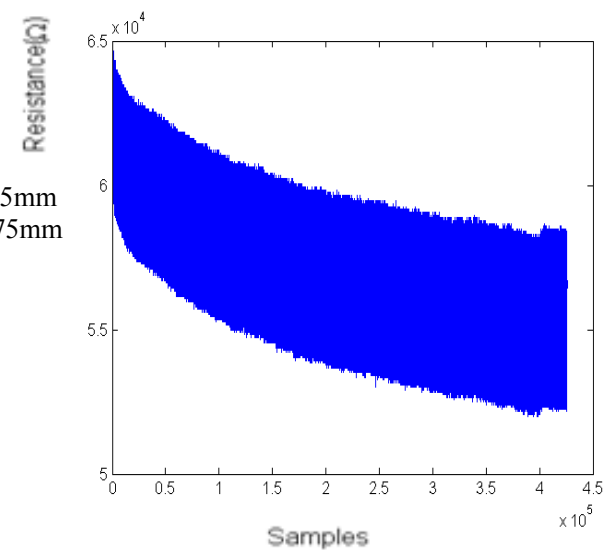

Figure 5. Response of the Black Sensor for a period of two and a half hours.

Lenght: $55 \mathrm{~mm}$

Width: $20 \mathrm{~mm}$

Textil sensor : named 2 Grey

Initial length material: $66 \mathrm{~mm}$

Final length material $=76 \mathrm{~mm}$

Extension: $10 \mathrm{~mm}$

Sample: 50 sps

Time test: $2,5 \mathrm{~h}$

Frequency: $0.142 \mathrm{hz}$

Total cycles: 1200

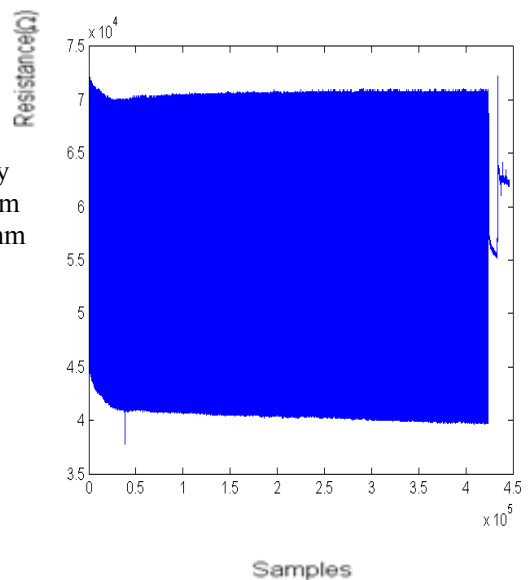

Figure 6. Response of the Grey Sensor for a period of two and a half hours.

\section{DISCUSSION}

The three different sensors proved adequate for monitoring respiratory rate. Although the Black sensor showed some drift in resistance over time the difference between the maximum and minimum resistance in each cycle remained constant.

Fig. 7 shows an example of the pre-tension problem in Grey sensor when the initial extension (in this case 10\%) is not enough and consequently, the signal is subject to considerable artefact noise. In case of the Grey sensor this effect is bigger and more difficult to remove without the use of the Butterworth filter.


cycles: 7

Figure 7a. Raw signal received from Shimmer platform. Figure $7 \mathrm{~b}$. Signal after application of Butterworth filter.

\section{CONCLUSIONS}

By characterizing the different response of each sensors material, we may progress to involve multiple sensors to examine the behavior of the whole thoracic and abdominal cavity. The diaphragm is the main inspiratory muscle, but other muscles involved in ventilation include the intercostal muscles and abdominals.

To obtain information about movement of the thoracic abdominal cavity during breathing, a new platform is being developing capable of saving data from 6 or more sensors while at the same time measuring body movements. That platform has a total of $11 \mathrm{ADC}$ that will permit to measure data from different piezoresistive sensors at the same time and other kind of sensors like temperature, humidity, etc.

Validation of the sensors will involve comparison with a gold standard $\mathrm{VO}_{2}$ max machine or spirometer. The effects of temperature also need to be investigated to ensure wearability and ruggedness of the sensors. All of these will permit detection of anomalies during respiration such as coughing. The multi-sensor vest (figure 8) will permit an answer trying to response why people have different problem related to respiration or trying to improve the rendiment in sports. 


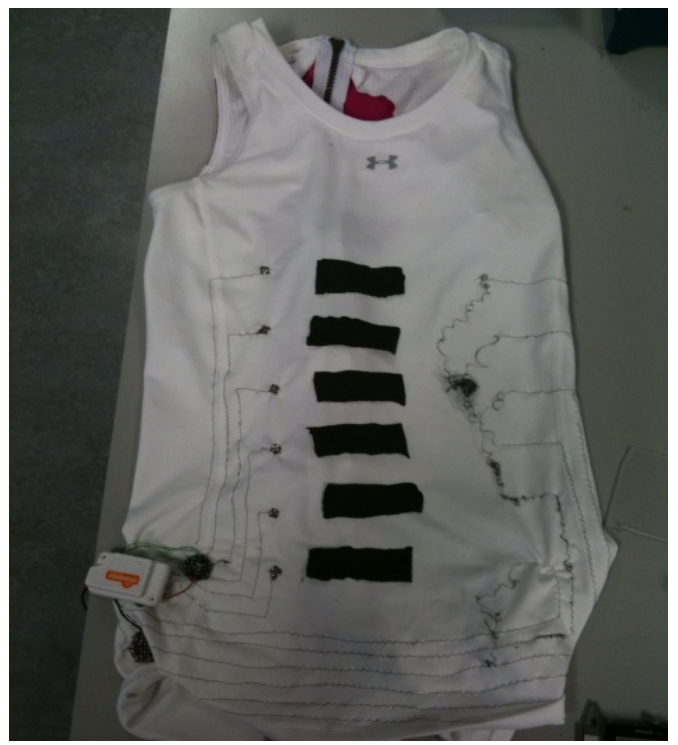

Figure 8. New shirt with 6 sensors connected to Shimmer platform.

\section{ACKNOWLEDGMENT}

This work is supported by IRCSET and Realtime Technologies under the Enterprise Partnership Scheme and Science Foundation Ireland under grant no. 07/CE/I1147.

\section{REFERENCES}

[1] Guenette, J. A. \& Sheel, A. W. (2007) Physiological consequences of a high work of breathing during heavy exercise in humans Journal of Science and Medicine in Sport, vol. 10, no., pp.341-350.

[2] Mitchell, E., Coyle, S., Ward, T., O'Connor, N. E. \& Diamond, D. (2010) Breathing Feedback System with Wearable Textile Sensors. Body Sensor Networks (BSN 2010) June 7 - 9, 2010, Biopolis, Singapore.

[3] Lymberis A, P. R. (2008) Smart fabrics and interactive textile enabling wearable personal applications: R\&D state of the art and future challenges. IEEE Eng Med Biol Soc.

[4] Shimmer Research, www.shimmer-research.com. 